Recepción: 20 / 04 / 2017

Aceptación: 20 / 05 / 2017

Publicación: 15 / 09 / 2017
Ciencias de la computación

Artículo Científico

\title{
Los Cursos Online Masivos Abiertos (MOOC) como una oportunidad para las PYMES en sus procesos de capacitación
}

Open Massive Online Courses (MOOC) as an opportunity for small and mediumsized enterprises (PYMES) in their training processes

\section{Open Mass Online Courses (MOOC) como uma oportunidade para as PME em seus processos de treinamento}

\author{
Marjorie Marcela Acosta Veliz ${ }^{\text {I }}$ \\ marjorie.acostav@ug.edu.ec \\ Verónica Coronel II \\ veronica.coronelpe@ug.edu.ec \\ Marco Navarrete III \\ marco.navarretep@ug.edu.ec
}

Correspondencia: marjorie.acostav@ug.edu.ec

I. Magíster en Administración y Dirección de Empresas, Ingeniera Comercial con Especialidad en Finanzas, Especialista en Evaluación de Proyectos y Gestión Directiva, Docente de la Universidad de Guayaquil, Guayaquil, Ecuador.

II. Magíster en Administración y Dirección de Empresas, con Especialidad en Finanzas y mención en Gestión empresarial. Docente de la Universidad de Guayaquil, Ecuador.

III. Magíster en Educación Superior, Ingeniero Comercial con Especialidad en Finanzas, Experiencia en Ventas consumo masivo, Docente de la Universidad de Guayaquil, Ecuador. 


\section{Resumen}

La presente investigación tuvo como objetivo mostrar la importante de los Cursos Online Masivos Abiertos en el currículo del trabajador actual como complemento a los amplios estudios en el campo educativo y su influencia en la educación de la sociedad actual. En los negocios y en el desarrollo empresarial presentan un gran aporte al momento de contratar personal y definir características que se buscan en el empleado moderno y competitivo para un sector tan importante como las PYMES. Se utilizará una metodología de investigación de documental, también se realizó investigación de campo a través de la realización de encuestas. Como resultado se obtuvo un gran desconocimiento de la tendencia que se marca a nivel mundial, pero también mucho interés en implementarlo en sus planes de capacitación y como parte de los factores de contratación laboral de nuevos elementos para la organización.

Palabras claves: Aprendizaje, Conocimiento, Empresa, MOOC, PYMES. 


\begin{abstract}
The present research aimed to show the importance of the Open Massive Online Courses in the curriculum of the current worker as a complement to the extensive studies in the field of education and its influence on the education of today's society. In business and in business development they present a great contribution when hiring staff and defining characteristics that are sought in the modern and competitive employee for a sector as important as PYMES. A documental research methodology will be used, also field research was carried out through the conduct of surveys. As a result, there was a great lack of awareness of the trend marked worldwide, but also a great deal of interest in implementing it in its training plans and as part of the recruitment factors of new elements for the organization.
\end{abstract}

Key words: Learning, Knowledge, Company, MOOC, PYMES. 


\section{Introducción.}

La tecnología ha abierto las puertas hacia un abanico de nuevos productos y servicios para uso del ser humano y con ello también la nueva oferta académica de medios para educarse. Uno de los grandes cambios revolucionarios se manifiesta en la educación ya que se han ampliados las diversas formas de adquisición y difusión de conocimientos, estructurando una nueva forma en que el docente imparta la cátedra hoy y en el futuro ya que es la tendencia que marca la historia del mundo moderno. Mengual, Vásquez y Eloy (2016) indicaron que cada vez se ofrece nuevas actividades de aprendizaje y los cursos y seminarios se crean y diseñan basados en el uso de la tecnología. A partir de esta corriente se desarrollan MOOC, es el acrónimo en inglés de Massive Online Open Courses (o cursos online masivos y abiertos), con el surgimiento del internet la educación ha visto nacer nuevos procesos y formas de impartir conocimiento. La página MOOC (2017), indicó que más de 700 universidades todos los meses presentan una oferta académica nueva y lo único necesario son una buena conexión de internet y ganas de aprender.

Learning (2017) manifestó que los MOOC tienden a hacerse más populares y las universidades de todo el mundo ven ellas una nueva oportunidad y se adhieren a la tendencia, obviamente es más notorio en países muy desarrollados, pero su principal características justamente es llegar a millones de personas alrededor del mundo a través de un computador, celular o Tablet. El acceso a internet de gran parte de la población ha permitido que este creciente impulso hacia las MOOC permita que cada vez más personas puedan acceder al conocimiento y nutrirse de nuevas experiencias en el constructo de sus nuevos conocimientos.

En los actuales momentos se procura que la educación logre estar al alcance de todos en condiciones de calidad para todos quienes tengan deseos de superarse y enriquecer sus carreras 
profesionales. En este contexto se pertinente investigar las tendencias en Guayaquil, la cual se constituye en una de las ciudades más importantes del Ecuador a través de la reflexión de cuán importante es un MOOC para el currículo, para el desarrollo personal y para asegurar un crecimiento profesional en un mundo tan competitivo.

\section{Definiciones}

En la época actual la sociedad está inmersa en un amplio espectro de datos e información que se transforma día con día. Los individuos cada vez encuentran nuevas y mejores formas de prepararse y consolidar sus conocimientos. Como indican (Jiménez, Acosta, \& Salas, 2017) "las oportunidades que ofrece la tecnología son infinitas, los aplicativos disponibles, los sitios de internet, promueven que exista una capacitación constante, con mínima inversión y hasta gratuita siempre al alcance de la persona interesada y ávida de conocimiento”.

Bartolomé y Steffens (2015) citaron que “ cuando Stephen Downes y George Siemens atendían la conferencia Desire21Learn, cansados de discutir las aplicaciones del conectivismo, pensaron que el mejor modo para comprender como funciona el aprendizaje en línea era crear un curso de este tipo", los Massive Open Online Courses, se definen con las siglas MOOC, siendo "una de las características que presentan números muy elevados de participantes y al ser cursos abiertos, los aprendices proceden de diversas nacionalidades” (Heinsch y Rodríguez, 2015, p. 2).

De acuerdo a Cabero et al. (2014). Las principales características del curso son:

- "Es un recurso educativo que tiene cierta semejanza con una clase, con un aula.

- Con fechas de comienzo y finalización.

- Cuenta con mecanismos de evaluación. 
- Es online.

- De uso gratuito.

- Es abierto a través de la web, y no tiene criterios de admisión.

- Permite la participación interactiva a gran escala de cientos de estudiantes. (Castaño

- y Cabero, 2013, 89).”

En la Figura 1, la nube de palabras recoge la terminología utilizada alrededor de la tendencia MOOC y que es cada vez más común utilizar en el ámbito educativo.

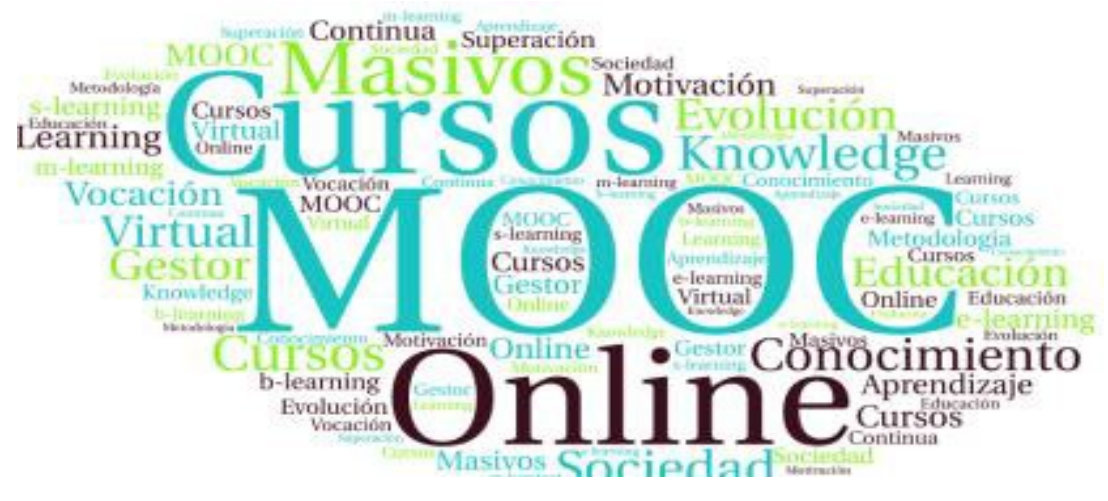

Figura 1 Nube de Palabras Nota: Elaborado en Software WordArt

Conole (2016) indicó que Siemens et al. creó el primer MOOC en 2008, llamado 'Conectivismo y conocimiento conectivo'. El curso fue basado en una pedagogía conectivista, cuyo objetivo era fomentar la participación. Se basó en los beneficios de la escala aunque interacción significativa con una red distribuida de pares. Fue un éxito rotundo, de allí se fueron generando nuevos modelos como el xMOOCs. Se basaron en medios interactivos tipo conferencias, videos y texto. Estos adaptaron un enfoque pedagógico conductista, haciendo énfasis en el aprendizaje individual. De estos modelos surgieron varias empresas como EdX, y Coursera, en las cuales los cursos son impartidos por Universidades de prestigio como Harvard y Stanford. Fidalgo et al (2013) 
indicó que con el uso de la tecnología, la estrategia didáctica y el nivel de cooperación se puede definir dos tipos de MOOC: los XMOOC y los cMOOC

Para García-Peñalvo y Seoane Pardo (2015) "lo que realmente aportan los MOOC para convertirse en el catalizador de un cambio en la formación online viene de la conjunción de, en primer lugar, la idea de la tecnología ... y, por otra parte, la idea del conocimiento distribuido", definiendo así la importancia de los MOOC en la sociedad actual, pues al ser un detonante, permite múltiples aplicaciones de este novedoso concepto de formación.

"La gratuidad y masividad son los dos conceptos que diferencian este tipo de formación del ya tradicional e-learning. Para que este movimiento siga avanzando precisa de una reconceptualización que supere la moda pasajera y genere un modelo didáctico sostenible”. (Aguaded-Gómez, 2013, p. 8).

Según Cabero (2015) se presume que los MOOC transformarán radicalmente la enseñanza existe una visión de que los MOOC será una tecnología que transformará y mejorará radicalmente la enseñanza, sin dejar de lado que se presente como una tecnología emergente en el entorno educativo y se debe conceptualizar que no por ser emergente es nuevo, más bien es una nueva forma de aplicación de la tecnología existente.

\section{Evolución}

De acuerdo a las estadísticas Shah (2016) en su reporte By the Numbers informó Las principales plataformas de MOOC en el mundo reúnen más de 700 universidades en el mundo, al finalizar el año 201658 millones de estudiantes estaban inscritos y cerca de 7000 cursos fueron impartidos y muchos países tienen sus plataformas propias como por ejemplo India, Mexico, Thailand, Italy, y otros. Entre las más importantes se destacan las de la Figura 2. 


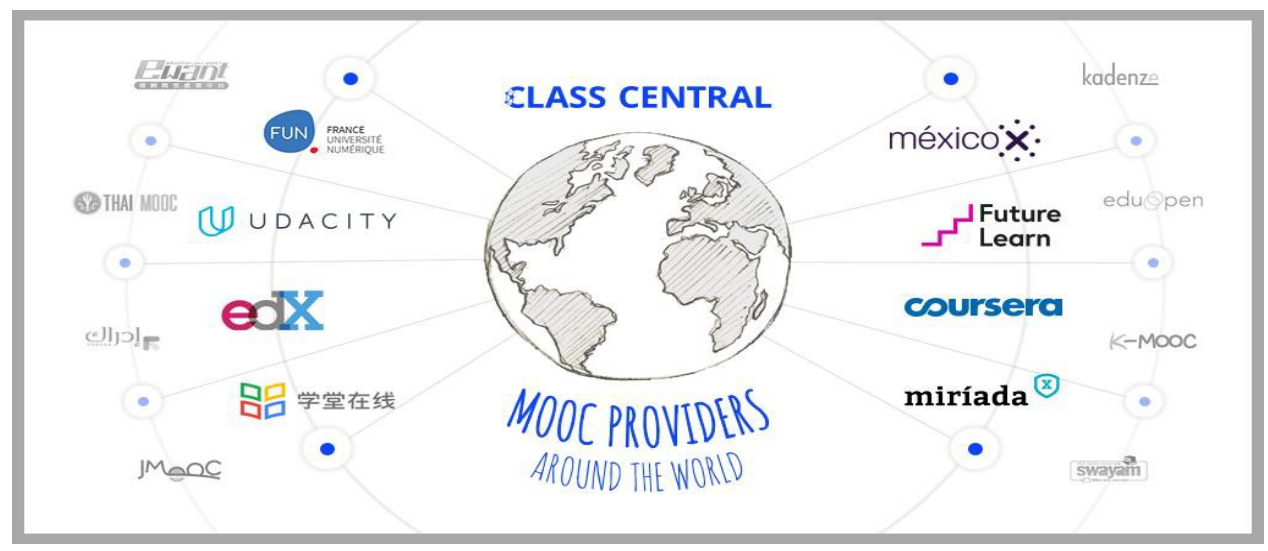

Figura 2 Plataformas en el Mundo

Fuente: Tomada de Shah (2017)

Un aspecto importante del crecimiento de los cursos es su oferta académica, en la Figura 3 se puede apreciar la variedad y peso de cada materia en la cantidad de cursos que se imparten, la cual va relacionada con cursos de Negocios y Tecnología (Informática, Ciencias de la Información, Programación) La categoría de negocios creció un 3\% este año y ese dato forma parte esencial del desarrollo del tema, puesto que cada vez es más importante en el ambiente de los negocios prepararse de forma autónoma para seguir creciendo.

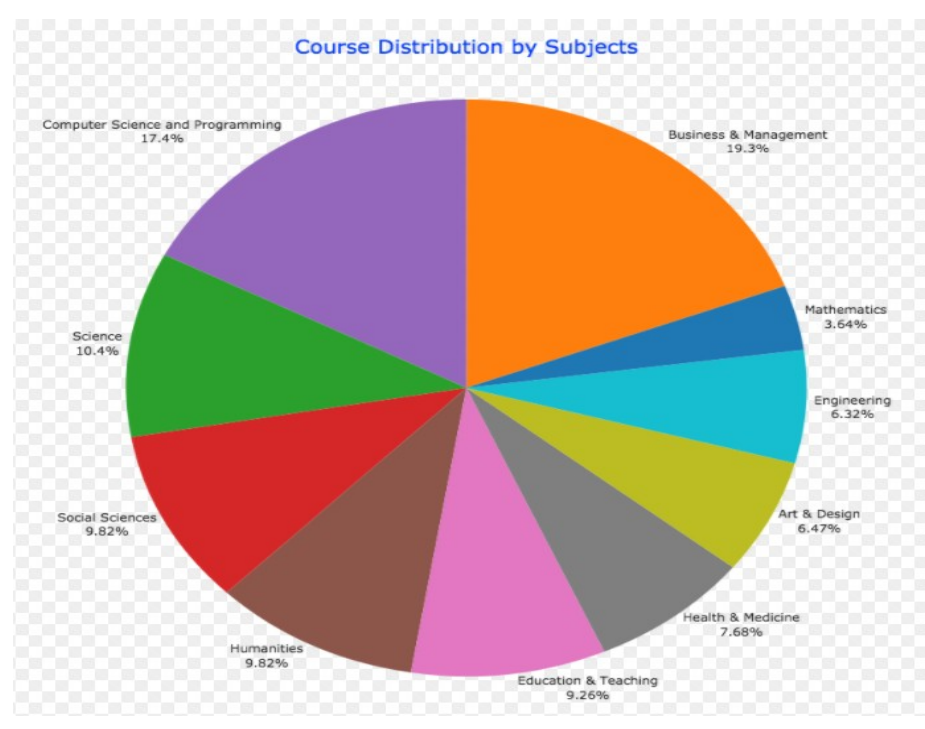

Figura 3 Oferta Académica Fuente: Tomada de Shah (2017) 


\section{MOOC en el Ecuador}

En Ecuador también esta tendencia a nivel educativo tiene sus exponentes y muchos cursos se han diseñado de acuerdo a la realidad del país, existen varias universidades que ofertan MOOC y en la Tabla 1 se enuncian sus enlaces y principales ofertas académicas.

\section{Tabla 1}

\section{Oferta Académica del Ecuador}

\begin{tabular}{|c|c|c|}
\hline Instituciones & Cursos MOOC & Página Web \\
\hline \multirow{5}{*}{$\begin{array}{l}\text { Universidad Particular } \\
\text { de Loja (UTPL, 2017) }\end{array}$} & Realidad Nacional & \multirow[t]{5}{*}{ https://mooc.utpl.edu.ec/ } \\
\hline & Técnicas de Expresión Oral y & \\
\hline & Escrita & \\
\hline & Metodología de Estudio & \\
\hline & Relaciones Públicas y Protocolo & \\
\hline \multirow{7}{*}{$\begin{array}{l}\text { Escuela Politécnica } \\
\text { Nacional (EPN, 2017) }\end{array}$} & Ofimática Básica & \multirow[t]{7}{*}{ http://mooc.virtualepn.edu.ec } \\
\hline & Google Drive & \\
\hline & Prezi & \\
\hline & Marketing de Contenidos & \\
\hline & De la Idea a un Modelo de & \\
\hline & Negocios Startup & \\
\hline & $\begin{array}{l}\text { Administración Básica de } \\
\text { Moodle }\end{array}$ & \\
\hline \multirow{3}{*}{$\begin{array}{l}\text { Escuela Superior } \\
\text { Politécnica del Litoral } \\
\text { (ESPOL, 2017) }\end{array}$} & Formulación de Objetivos en & \multirow[t]{3}{*}{ https://www.virtual.espol.edu.ec/courses } \\
\hline & Educación, Procesos Didácticos & \\
\hline & Inglés, entre otros & \\
\hline
\end{tabular}

Estas tres universidades ofrecen gratuitamente la preparación y en el caso de la Escuela Politécnica Nacional ofrece la certificación física por un valor económico. Los cursos están diseñados para que pueden ser tomados por cualquier usuario y su desarrollo demanda entre 10 y 50 
horas de esfuerzo para su aprobación, dependiendo del ritmo de estudio del estudiante. En la UTPL, los cursos pueden acreditarse para aprobación de materia del semestre, validando sus conocimientos con un examen presencial en la sede y cancelando el valor respectivo.

Finalmente en lo referente a formación y capacitación de los servidores públicos, el Ecuador se encuentra en un desafío si se toma en cuenta que solamente la Función Ejecutiva consta de 479.993 servidores (según datos del sistema de pagos del Ministerio de Finanzas) y existen 6.119 instituciones públicas en todo el país (de acuerdo con el catastro del Ministerio de Relaciones Laborales). De ahí que acudir a la tecnología deja de ser una opción para volverse una necesidad imperiosa para lograr que el conocimiento especializado en cada área pueda ser difundido (Telégrafo, 2014). A cargo de esto se encuentra el Instituto de Altos Estudios Nacionales (IAEN) que trabaja desde mediados de 2013 en la adaptación y traducción del código fuente de la tecnología de Cursos en Línea Masivos y Abiertos (MOOC, por sus siglas en inglés), a través de la plataforma digital EDX, utilizada por las más prestigiosas universidades del mundo, desde Harvard hasta la UNAM. Este, que es el producto estrella de su departamento de e-learning, permitirá revolucionar tanto cuantitativa como cualitativamente la capacitación y formación de miles de servidores públicos de todo el Estado, a un costo mucho menor y con un alcance ilimitado, estos cursos actualmente ya están disponibles en su página de internet (IAEN, 2017).

\section{MOOC en los negocios}

En el contexto educativo hay muchísimas aplicaciones pero y en los negocios? Según González (2017) "los MOOC (Massive Open Online Courses) están cambiando dos ámbitos: la universidad y la empresa. Es una de las conclusiones de la Conferencia Internacional de MOOC que se celebró en mayo en Madrid en la Universidad Carlos III”. Dos aspectos básicos del entorno 
empresarial son la formación en su índole interna y la percepción que tiene el empleador con respecto a esta preparación al momento de contratar a un nuevo trabajador.

Aunque todavía no se hace parte de la entrevista de trabajo, poco a poco ha tomado importancia que se destaque la preparación fueras de las aulas, ya que cada vez más se requiere destacar esas competencias al solicitar un puesto de trabajo, potenciar los conocimientos tecnológicos, motivación y la responsabilidad que demandan estos tipos de cursos para culminarlos con éxito.

Como un dato importante la plataforma Miríada X "ha impartido más de 600 Mooc de 27 temáticas, promediando 5.000 alumnos por curso y una tasa de finalización del $23 \%$, una de las más elevadas, ya que la media mundial se sitúa entre el 8\% y 10\%”(Otiniano, 2017). Esta oferta académica es mayoritariamente en español, por tanto es clave al momento de catalogar la participación de Ecuador en este tipo de cursos y en la Figura 4 se muestra la estadística de del país en esta plataforma y en la cual se evidencia la preferencia por cursos de tecnología y Big Data.

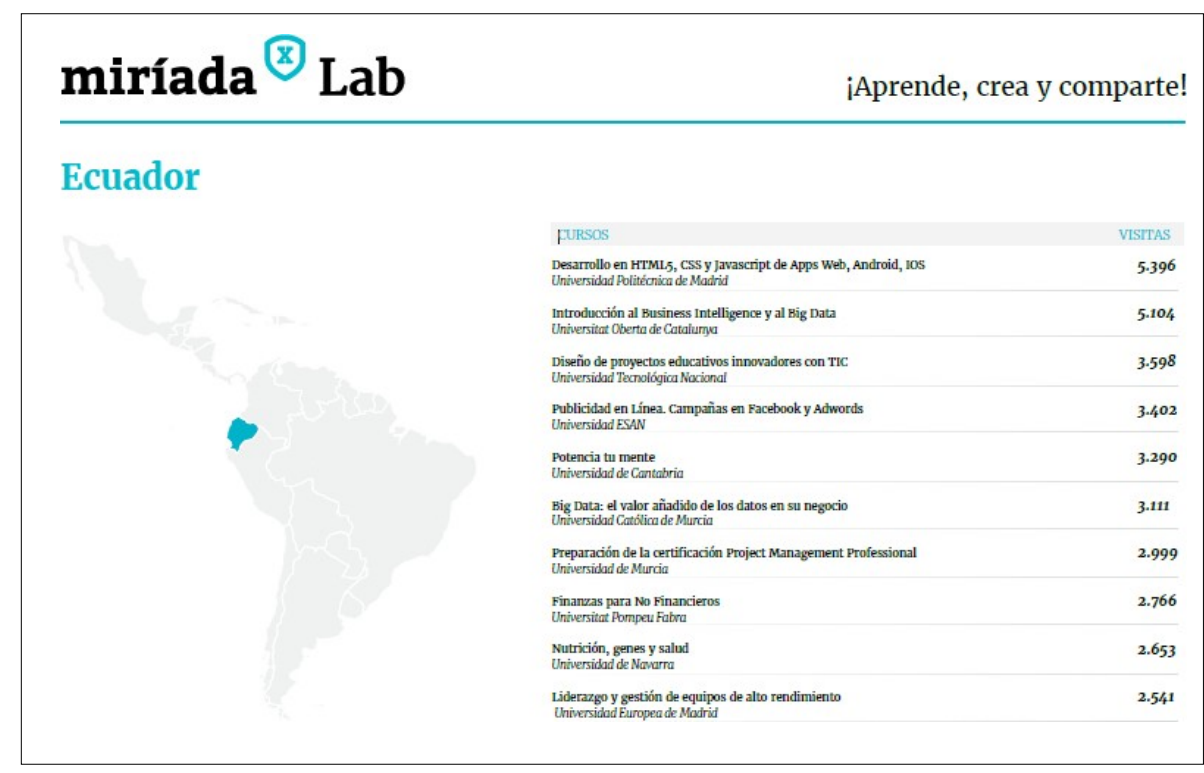

Figura 4 Estadísticas del año 2016 Ecuador

Fuente: Tomado de (Miríadax, 2017) 
Varias plataformas han incursionado en cursos con especialidades directas para desempeñar puestos de trabajo específicos, algunas de ellas tienen como socios estratégicos, por ejemplo EDX, cuenta con Microsoft y en sus ofertas de cursos se vincula la formación profesional diferenciada para cubrir necesidades laborales de esa índole, proporcionando no sólo cursos, sino Programas, Micromasters y XSeries, los cuales "han sido desarrollados por especialistas de renombre mundial y por universidades de prestigio y están diseñados para proporcionar un conocimiento profundo sobre temas fundamentales a través de una serie de cursos". (EDX, 2017).

En la plataforma Miríada X, se trabaja con un comité de expertos MOOC, generando "una plataforma de encuentro de profesionales de diferentes industrias, organizaciones y países que trabajan para avanzar en el desarrollo del aprendizaje social a través de Cursos masivos abiertos online (MOOCs)" (Miríadax, 2017) buscando satisfacer necesidades específicas para las tendencias de los cursos y la generación de nuevas demandas.

Udacity es una página pionera en los nanocursos o Nanodegrees como se denominan en su página, son cursos y programas directamente diseñados bajo requerimientos o en colaboración con Google, AT\&T, Amazon, Facebook, IBM, BOSC, Didi, Hootsuite, entre otras pudiendo llegar a 6 meses de preparación. Sus ámbitos se relacionan con tecnología, robótica, marketing, realidad virtual y muy variada oferta sobre data science según su página web (UDACITY, 2017).

Siendo estas las plataformas más importantes a nivel mundial, reflejan ya la tendencia de adaptar la capacitación a las necesidades actuales de los negocios, facilitando al usuario, recibir cursos de formación especializada y en concordancia a sus requerimientos de conocimiento para el desenvolvimiento de sus actividades profesionales, principalmente en las esferas tecnológicas, análisis de datos, inteligencia artificial, robótica, nanotecnología, etc. Es válido señalar un aspecto importante resaltado por Ruiz e Ithai (2016) "la evaluación en los MOOC 
requiere comenzar a pensar dinámicas propias en las que el estudio de los números nos dé no una tendencia sino un significado cualitativo del progreso de cada persona" y es allí donde la aplicación del conocimiento en la práctica consolida la evaluación, puesto que en la masificación de los cursos es difícil llegar a conocer la profundidad del conocimiento logrado, pero si la persona logra llevarlo a la práctica, su aprendizaje se ve puesto a prueba en el mercado laboral y profesional.

Las aprobaciones de un MOOC reflejan características importantes que buscan los trabajadores de hoy en día "capacidad de superación, esfuerzo, creatividad y vocación por el aprendizaje permanente" (Otiniano, 2017). "El rejuvenecimiento de la productividad se convierte en una condición necesaria para permanecer actualizado.” (UniMOOC, 2012). Los empleadores deberán aprovechar todas estas facilidades que brinda la potencialidad del uso de las tecnologías de información para facilitar la capacitación de sus trabajadores. La cultura de norteamérica y europea ya potencia esta preparación, en los países de América Latina y el Caribe ya están incursionando en estos estilos.

En Latinoamérica existe una poderosa iniciativa de parte de Fundación Carlos Slim, a través de Capacítate para el Empleo, en la cual existe una oferta de cursos (más de 100 disponibles) inclusive Diplomados de temas relacionados con Comercio Digital, Industria, Energía, Construcción, Moda y Belleza, Salud, Transporte, Tecnología, etc., los cuales permiten a los participantes obtener certificaciones online y en el caso de Diplomados una certificación oficial del Gobierno de los Estados Unidos Mexicanos, que se envía a los domicilios de los estudiantes que aprobaron. Es una página muy interactiva con más cursos listos para ser abiertos a los interesados y permite aprendizaje en varias aristas de conocimiento. Con ello buscar fortalecer a quienes participan y brindarles la oportunidad de participar en una bolsa de trabajo. La oferta completa de curso está disponible en su página web" Capacítate para el Empleo" (FundaciónCarlosSlim, 2017). 


\section{Metodología}

Se utilizará una metodología de investigación de documental como revisión bibliográfica, estadística, etc. También se realizó investigación de campo, siendo esta una excelente herramienta para soporte de resultados de otras informaciones generadas en entrevistas, encuestas, cuestionarios e incluso observaciones. Se incluye la investigación descriptiva la cual de acuerdo Marín (2008) logra caracterizar un objeto de estudio o una situación concreta, señalar sus características y propiedades.

El estudio se basa en los métodos inductivo y deductivo, la técnica a usar será la encuesta, la cual se realizó a varias PYMES de algunos sectores de negocios de la ciudad de Guayaquil a los cuales se tuvo acceso, totalizando 20 , como un sondeo preliminar, clasificada como se muestra en la Tabla 2.

\section{Tabla 2}

\section{Encuestados por Sector}

SECTOR PYMES NÚMERO DE ENCUESTADOS

\begin{tabular}{lc}
\hline Alimentos & 4 \\
Siderurgia & 4 \\
Bebidas & 4 \\
Comidas Rápidas & 4 \\
Construcción & 4 \\
\hline
\end{tabular}




\section{Resultados}

Se realizaron tres preguntas claves para conocer la opinión del personal encargado de la contratación y capacitación de las compañías participantes.

\section{Pregunta 1}

¿Conoce qué es un MOOC? La Figura 5 refleja los resultados de la interrogante y deja en evidencia el desconocimiento de lo que representa un MOOC, solo dos personas conocían por alguna mención dentro de algún programa de capacitación, pero no han tenido la oportunidad de desarrollar ninguno.

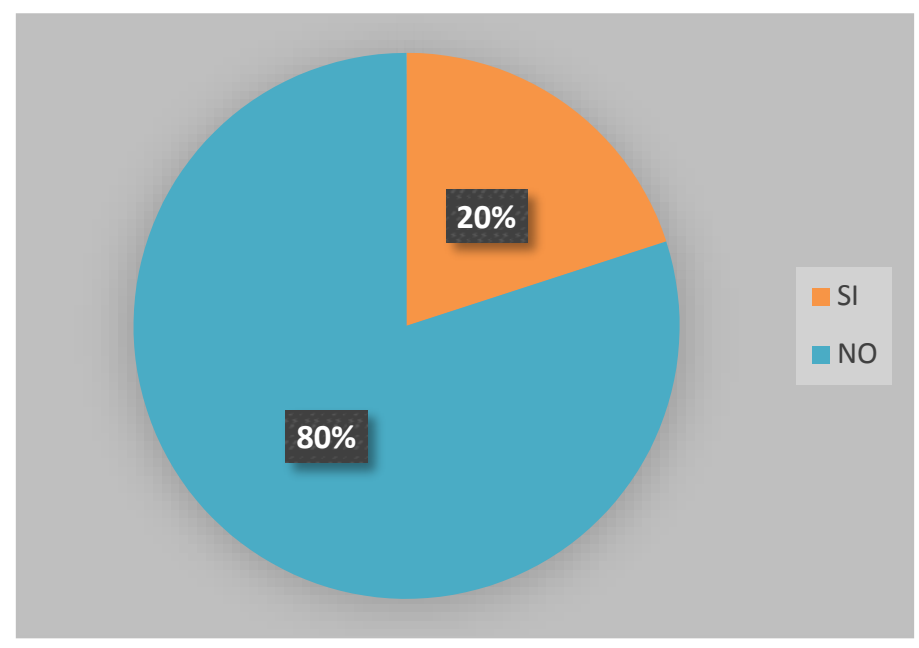

\section{Figura 5 ¿Conoce qué es un MOOC?}

\section{Pregunta 2}

¿Tomaría en consideración al momento de contratar personal la experiencia y certificación del trabajador en cursos MOOC? Una vez explicado el concepto se procedió a realizar la pregunta y los resultados reflejan un potencial interés en tomar en consideración este aspecto, fortaleciendo que esta característica proporciona más datos de la superación y motivación personal hacia aprender más de los potenciales candidatos 


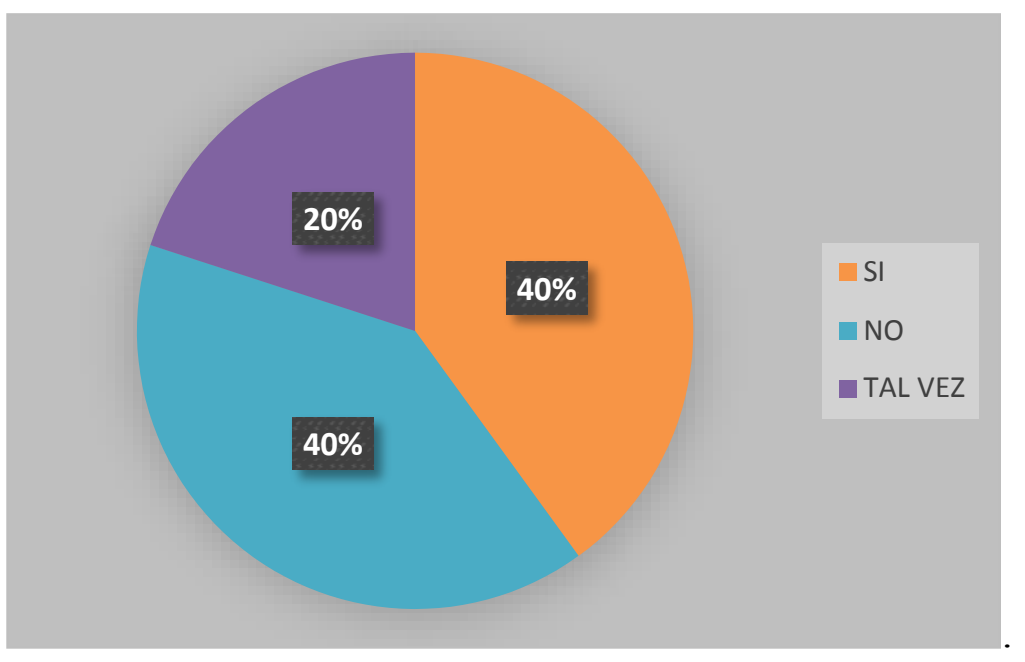

Figura 6 ¿Tomaría en consideración al momento de contratar personal la experiencia y certificación del trabajador en cursos MOOC?

\section{Pregunta 3}

¿Utilizaría los MOOC como parte de los planes de capacitación del personal? La Figura 7 presenta los resultados, en los cuales se evidencia que las empresas si están dispuestas a formar a sus trabajadores con los MOOC, ya que puntualizan que optimizan los costos de capacitación, ya que las certificaciones en la mayoría de los casos son gratuitas y en otros los costos son inferiores a los $\$ 100.00$. 


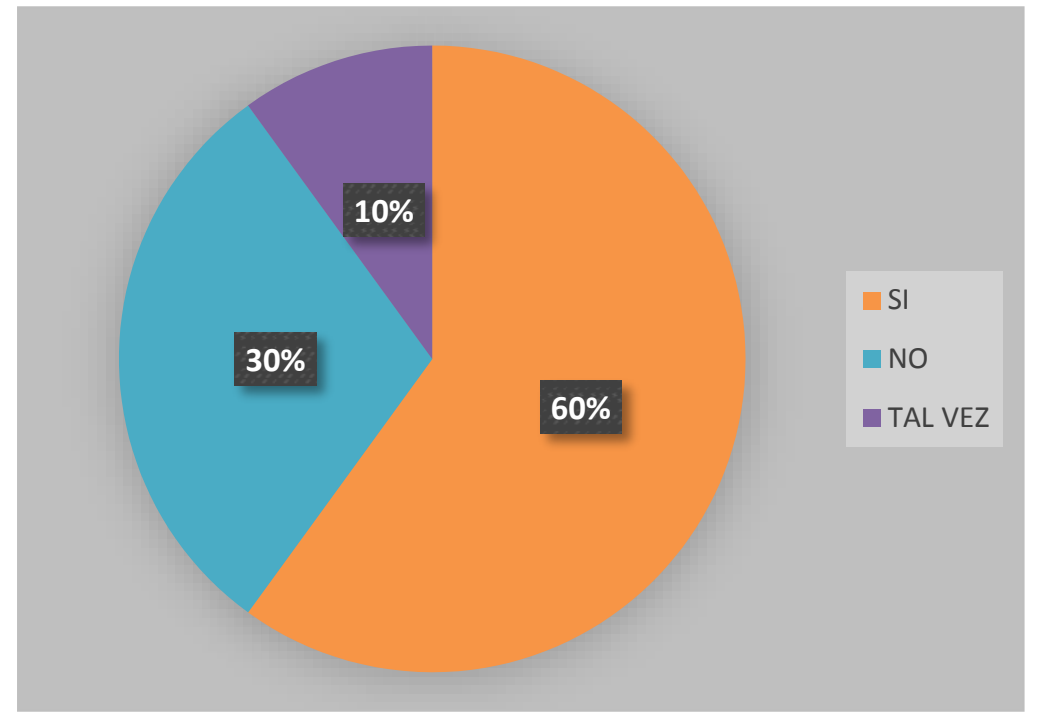

Figura 7 ¿Utilizaría los MOOC como parte de los planes de capacitación del personal?

Estas preguntas en esencia muestran la situación actual del potencial que pueden llegar a tener los MOOC en el desarrollo personal a nivel empresarial. La muestra representa ciertos sectores que tuvieron la apertura para conocer sobre MOOC e idear su inclusión dentro del desarrollo de sus negocios. Las PYMES son gran parte de los emprendimientos en el Ecuador y representen un mercado de amplio crecimiento.

\section{Conclusiones.}

La tecnología se constituye en una aliada para la educación y los negocios, no deben verse por separado, sino como una sinergia holística que cubre muchos de los aspectos del desarrollo del individuo y su realización personal, principalmente al momento de encontrar un empleo acorde a sus capacidades y potencialidades en el entorno laboral, el mismo que cada vez exige más del empleado y que su multifuncionalidad le permite obtener puestos de nivel jerárquico superior.

Es necesaria mucha difusión aún en las empresas locales, ya que la investigación solo en la ciudad de Guayaquil demuestra el desconocimiento presente sobre esta oportunidad de formación y capacitación de los prospectos de empleados y de los empleados que ya forman parte de la plantilla 
de trabajadores. La autoformación permite visualizar cualidades de una persona hacia el crecimiento y la superación, es una característica del learnability que en la sociedad del siglo XXI hace trascender la diferenciación entre trabajadores.

En un entorno competitivo y en economías con restricción, las empresas deben ver la capacitación de MOOC como un soporte a sus planes anuales de capacitación. Las ofertas académicas son amplias y cubren la preparación en gran cantidad de áreas empresariales desde administración, marketing, finanzas, producción, desarrollo web, sistemas ERP, análisis de Big Data, Responsabilidad Social y otros. Pueden realizarse estudios de prueba para probar su efectividad dentro de la organización, futuros análisis comprobarán los resultados de esta unión cooperativa del MOOC y el desarrollo de los profesionales en las empresas.

Las PYMES tiene una oportunidad potencial en el uso de MOOC, su disponibilidad ajustada de recursos posibilita su incursión en la preparación y capacitación de su personal, incluso otras.

\section{Bibliografía}

Aguaded-Gómez, J. I. (2013). La revolución MOOCs,i una nueva educación desde el paradigma tecnológico?/The MOOC revolution: A new form of education from the technological paradigm?. Comunicar, 21(41), 7-8.

BARTOlOMÉ, A.R. \& STEFFENS, K. (2015). ¿Son los MOOC una alternativa de aprendizaje? [Are MOOCs Promising Learning Environments?]. Comunicar, 44, 91-99. https://doi.org/10.3916/C44-2015-10

Cabero Almenara, J., Llorente Cejudo, M. D. C., \& Vázquez Martínez, A. I. (2014). Las tipologías de MOOC: su diseño e implicaciones educativas.

Cabero Almenara, J. (2015). Visiones educativas sobre los MOOC. RIED. Revista Iberoamericana de Educación a Distancia, 18(2).

Conole, G. (2016). Los MOOC como tecnologías disruptivas: estrategias para mejorar la experiencia de aprendizaje y la calidad de los MOOC. Revista de Educación a Distancia, (50). Información, 16(1).

EDX. (2 de Marzo de 2017). EDX. Obtenido de https://www.edx.org/xseries 
EPN. (15 de Marzo de 2017). Cursos de Autoestudio. Obtenido de http://mooc.virtualepn.edu.ec

ESPOL. (26 de Febrero de 2017). Virtual Espol. Obtenido de https://www.virtual.espol.edu.ec/

Fidalgo Blanco, Á., Sein-Echaluce Lacleta, M. L., Borrás Gené, O., \& García Peñalvo, F. J. (2014). Educación en abierto: Integración de un MOOC con una asignatura académica. Teoría de la Educación. Educación y Cultura en la Sociedad de la Información, 15(3).

FundaciónCarlosSlim. (1 de Abril de 2017). Capacitate para el empleo. Obtenido de https://capacitateparaelempleo.org/

García-Peñalvo, F. J., \& Seoane Pardo, A. M. (2015). Una revisión actualizada del concepto de eLearning. Décimo Aniversario. Teoría de la Educación. Educación y Cultura en la Sociedad de la Información, 16(1).

González, A. (6 de Junio de 2017). MiriadaX. Obtenido de https://miriadax.net/blog/-/blogs/losempleadores-y

Heinsch, Bárbara; Rodríguez Pérez, María de las Nieves (2015). MOOC: un nuevo escenario de enseñanza-aprendizaje de lenguas extranjeras. @tic. revista d'innovació educativa. ( $\left.\mathrm{n}^{\mathrm{o}} 14\right)$. UR1. Fecha de consulta, 01/05/2016.

IAEN. (21 de Abril de 2017). Educación Virtual IAEN. Obtenido de http://educacionvirtual.iaen.edu.ec/course/index.php

Jiménez, M., Acosta, M., \& Salas, L. (2017). Learnability como característica del ciudadano del siglo XXI. Revista Dominio de las Ciencias, 54-71. doi:http://dx.doi.org/10.23857/dom.cien.pocaip.2017.3.4.jul. 54-71

Learning, A. (7 de Abril de 2017). American Learning \& Media. Obtenido de http://www.americalearningmedia.com/component/content/article/539-entrevistas/7368modelo-aprendizaje-moocs-promueve-competencias-transversales-esenciales-

Marín, A. (7 de Marzo de 2008). Metodología de la Investigación. Obtenido de https://metinvestigacion.wordpress.com/

Mengual, S., Vásquez, E., \& Eloy, L. (2016). La productividad científica sobre MOOC: aproximación bibliométrica 2012-2016 a través de SCOPUS. RIED Revista Iberoamericana de Educación a Distancia, 39-58.

Miríadax. (18 de Abril de 2017). Miríadax. Obtenido de Miríadax: https://miriadax.net/blog//blogs/top-10-de-los-cursos-con-mas-interes-de2016 ?p_p_auth $=$ daEYXcS1\&_33_redirect $=$ https $\% 3 \mathrm{~A} \% 2 \mathrm{~F} \% 2 \mathrm{Fmiriadax} . n$ et $\% 2 \mathrm{Fmi}-$ pagina $\% 3 F p \_p \_i d \% 3 D 115 \% 26 p \_p \_l i f e c y c l e \% 3 D 0 \% 26 p \_p \_s t a t e \% 3 D$ Dnormal $\% 26 p \_p \_$mode $\% 3$ Dview\%26p_p_col_id\%3Dcolumn-2\%26p_p_co

Mooc.es. (18 de Abril de 2017). Mooc.es. Obtenido de Mooc.es: http://mooc.es/que-es-un-mooc/ 
Otiniano, C. (21 de Abril de 2017). Los empleadores comienzan a tomar en serio los Mooc. Obtenido https://cincodias.elpais.com/cincodias/2017/04/21/fortunas/1492792443_261951.html

Ruiz, B., \& Ithaí, I. (2016). Los MOOC en números, un análisis para comenzar la reflexión. Tema del mes.

Shah, D. (25 de Diciembre de 2016). By The Numbers: MOOCS in 2016. Obtenido de https://www.class-central.com/report/mooc-stats-2016/

Shah, D. (15 de Junio de 2017). Class Central. Obtenido de https://www.classcentral.com/report/mooc-providers-list/

Telégrafo, E. (4 de Febrero de 2014). Tecnología MOOC en el Estado ecuatoriano. Guayaquil, Guayas, Ecuador. Obtenido de http://www.eltelegrafo.com.ec/noticias/tecnologia/30/tecnologia-mooc-en-el-estadoecuatoriano

UDACITY. (1 de Enero de 2017). UDACITY. Obtenido de https://www.udacity.com/

UniMOOC. (2 de Octubre de 2012). Observatorio MOOC. Obtenido de http://blogmooc.iei.ua.es/2012/10/la-importancia-de-los-mooc-para-las.html

UTPL. (1 de Marzo de 2017). UTPL MOOC. Obtenido de https://mooc.utpl.edu.ec/ 